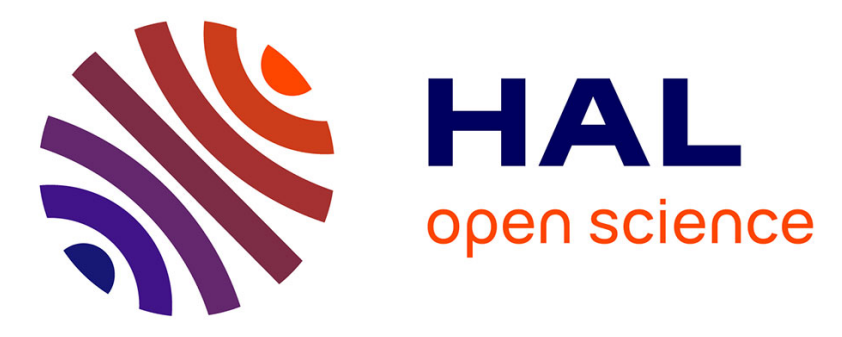

\title{
Analysis of RATAN-600 radiation patterns in new operation modes using the MLPO algorithm
}

\author{
Christine Letrou, M. K. Lebedev, Vladimir B. Khaikin, Amir Boag
}

\section{To cite this version:}

Christine Letrou, M. K. Lebedev, Vladimir B. Khaikin, Amir Boag. Analysis of RATAN-600 radiation patterns in new operation modes using the MLPO algorithm. COMCAS 2017: IEEE International Conference on Microwaves, Antennas, Communications and Electronic Systems, Nov 2017, Tel-Aviv, Israel. pp.1 - 3, 10.1109/COMCAS.2017.8244848 . hal-01686731

\section{HAL Id: hal-01686731 https://hal.science/hal-01686731}

Submitted on 17 Jan 2018

HAL is a multi-disciplinary open access archive for the deposit and dissemination of scientific research documents, whether they are published or not. The documents may come from teaching and research institutions in France or abroad, or from public or private research centers.
L'archive ouverte pluridisciplinaire HAL, est destinée au dépôt et à la diffusion de documents scientifiques de niveau recherche, publiés ou non, émanant des établissements d'enseignement et de recherche français ou étrangers, des laboratoires publics ou privés. 


\title{
Analysis of RATAN-600 Radiation Patterns in New Operation Modes Using the MLPO Algorithm
}

\author{
Christine Letrou ${ }^{1}$, Michael Lebedev $^{2}$, Vladimir Khaikin ${ }^{2}$, and Amir Boag ${ }^{3}$ \\ ${ }^{1}$ Télécom SudParis, Lab. SAMOVAR (CNRS UMR 5157), 91011 Evry Cedex, France \\ ${ }^{2}$ The Special Astrophysical Observatory, RAS, Russia \\ ${ }^{3}$ School of Electrical Engineering, Tel Aviv University, Tel Aviv 69978, Israel
}

\begin{abstract}
The multilevel physical optics (MLPO) algorithm has been adapted to compute radiation patterns of the triple reflector antenna of RATAN-600 radio telescope in new operation modes. These operation modes facilitate tracking of cosmic sources of radiation at arbitrary azimuthal angles in the range of $\pm 30^{\circ}$. The resulting radiation patterns are compared to those obtained via ray tracing analysis.
\end{abstract}

Index Terms - Radio astronomy, radiotelescope, reflector antennas, radiation pattern, physical optics.

\section{INTRODUCTION}

RATAN-600 radio telescope is a variable profile antenna, whose main reflector (also referred as "main mirror") consists of a number of panels, each capable to move in the radial direction and rotate around the azimuthal and elevation axes. The antenna system "South sector + Flat reflector" consists of the south sector of the main reflector, the flat reflector and the secondary reflector with the primary feed on the arc rail path (see Fig. 1). Such a configuration would allow tracking cosmic sources in a "running parabola" mode with a full aperture (of about 300 m) during 3 to 4 hours $\left(\alpha=0 \pm 30^{\circ}\right)$, when at each moment the main mirror would be a parabolic cylinder with an axis in the $-\alpha$ direction. This mode requires continuous modification of the antenna profile or at least switching between few thousands antenna settings per hour, which would be impractical with the existing automatic control system and drive mechanics, so it has not been realized up till now. Several methods were proposed to track cosmic sources using a fixed main reflector in the form of a circular cylinder. A secondary reflector of double curvature and a focal linear phased array [1] or the "radio-Schmidt" mode utilizing a distorted flat reflector [2] may be used for the correction of a spherical (cylindrical) aberration. In order to test the radio-Schmidt mode without distorting the flat reflector (which would be technically difficult), the longduration Sun tracking with a reduced aperture (of about $100 \mathrm{~m}$ ) was proposed and tested [3]. It was possible to track the Sun in the time interval of \pm 0.5 hour from meridian with acceptable aberrations. More advanced configurations allowing tracking cosmic sources not only near the meridian are described in the next section.

\section{ANTENNA CONFIGURATIONS IN NEW OPERATION MODES}

In this section we describe a novel antenna operating mode facilitating tracking the Sun not only near the meridian, but around any azimuth $\alpha$ to which the antenna is set. The simulation and the experiment show that the optimum continuous source tracking interval is $\delta \alpha=4^{\circ}$ $\left( \pm 2^{\circ}\right)$ or $16( \pm 8)$ minutes. As a result it may be possible to track the Sun with a reduced aperture $(100 \mathrm{~m})$ during 3 to 4 hours with 15 antenna settings only. Each of them includes main reflector setting at a given azimuth and flat reflector setting to compensate for the change in elevation of the cosmic source.

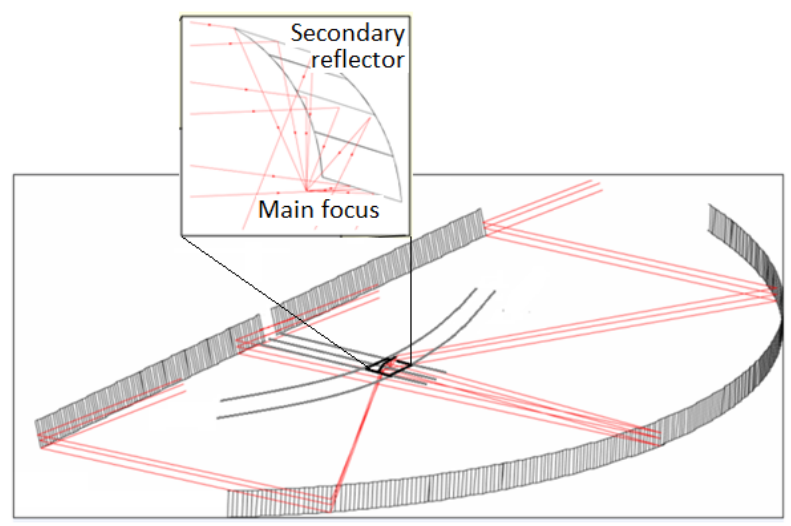

Fig. 1. "South sector + Flat reflector" antenna system.

Consider two settings of the main reflector:

- a parabola with a focal length of $131.8 \mathrm{~m}$, whose apex is $288.0 \mathrm{~m}$ away from the antenna center;

- a circle with the same focal length as that parabola, i.e. with a radius of $2 \times 131.8 \mathrm{~m}$; apex is at the same point.

Both shapes have the same radius of curvature and are close enough to each other. The aperture width is, say, $100 \mathrm{~m}$ in both cases (see Fig. 2).

The secondary reflector together with the primary feed can move along the circular rail path, whose center coincides with the center of the telescope. Its radius is chosen to ensure that if the axes of the main and the secondary mirrors coincide (axially-symmetric configuration) and the main reflector is set as a parabola, then the radiation from the main reflector after being 
reflected by the secondary reflector is focused exactly at the feed, i.e. the distance between the apices of the secondary and the main reflectors is $(131.8-2.15) \mathrm{m}$. In other words, the focal point moves along the circle with a radius of $(288-131.8+2 \times 2.15) \mathrm{m}$.

(a)

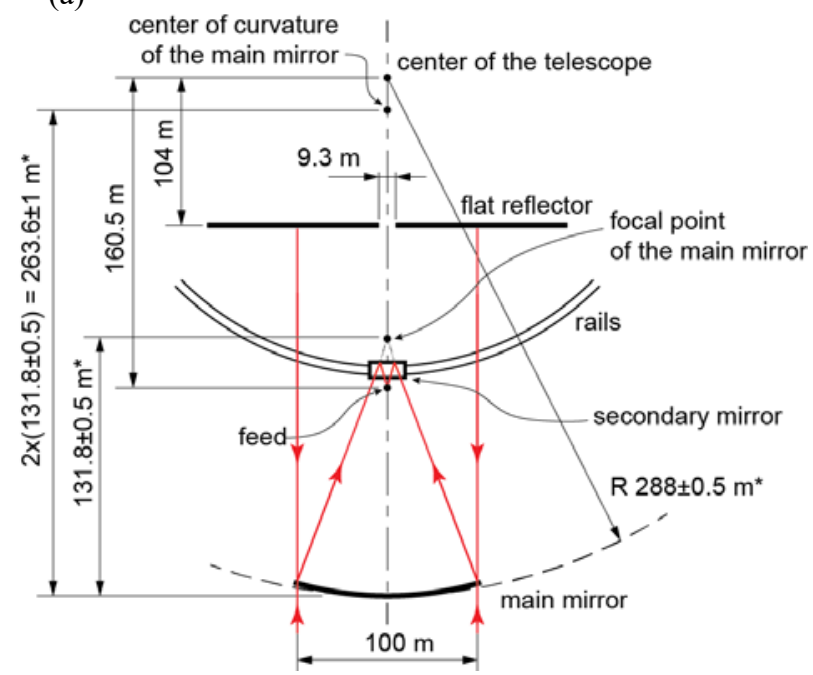

(b)

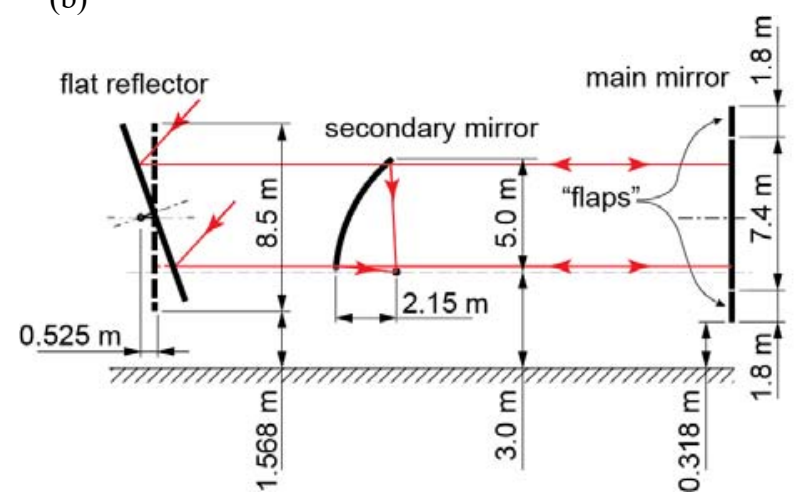

Fig. 2. Antenna set for a source at the meridian; (a) top view; (b) side view.

If the aperture of the main window, the feed and the secondary reflector are rotated around the center of the telescope by angle $\alpha$, then the only difference from the antenna settings for the source at the meridian is the mutual position of the flat reflector and the aperture, the secondary and the feed as a whole. In such a manner, one can observe the celestial source at the azimuth of $-\alpha$. Now, if we leave the aperture fixed and displace the secondary mirror along the rails by an angle $\delta \alpha$ from the axis, counting from the antenna center (Fig. 3), the maximum of the antenna radiation pattern will shift in the opposite direction.

If the telescope was designed with an arc rail path just at a halfway between the main mirror and the telescope center, there would be a rotational symmetry in the configuration with a circular main reflector, since for any $\delta \alpha$ the mutual position of the main reflector surface and the secondary reflector would be the same. However, in the present configuration the center of the telescope and of the arc rail path do not coincide with the center of curvature of the main reflector, hence as $\delta \alpha$ increases, the secondary reflector tends to get closer on its path to the main mirror. This is a reason of an additional aberration which grows when $\delta \alpha$ increases, but it is possible to mitigate the fall of the antenna gain vs. $\delta \alpha$ by optimizing the radius of curvature of the main reflector with the reduced aperture.

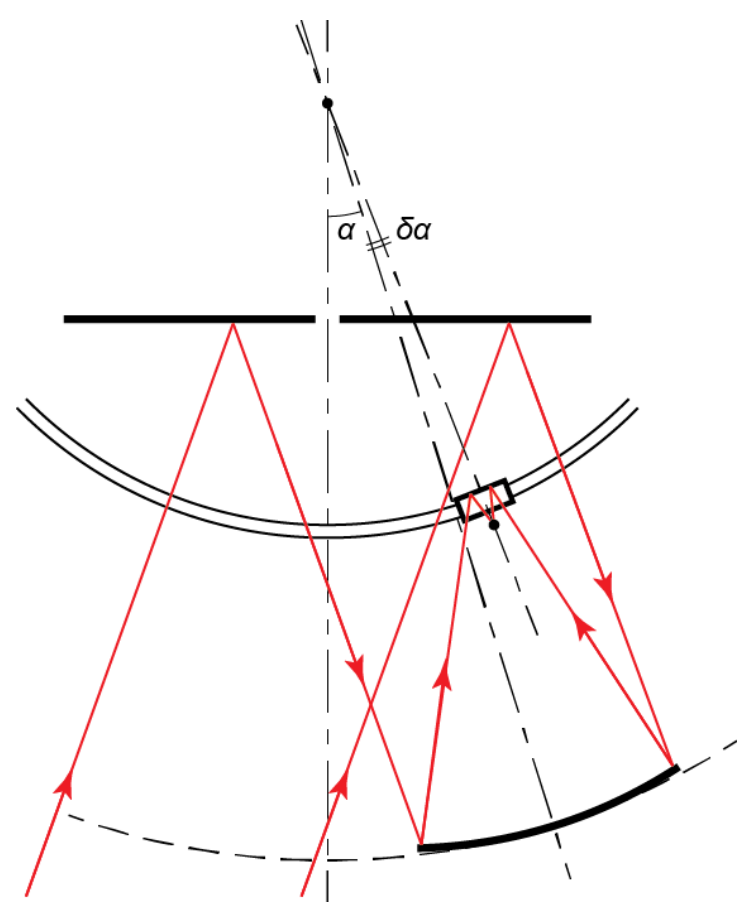

Fig. 3. Antenna set at azimuth $\alpha$, with secondary mirror displaced by an angle $\delta \alpha$.

\section{MLPO ANALYSIS}

The MLPO algorithm, originally developed in [4], is based on the observation that small radiating apertures produce smooth radiation patterns, which can be sampled on very coarse grids and fully reconstructed by interpolation. The MLPO algorithm starts with a hierarchical decomposition of radiating surfaces into small patches. The patterns of these small patches are calculated on a common coarse grid, in patch-centered coordinate systems. In these systems, the phase variations of the fields are slow, allowing for pattern interpolations with prescribed accuracy. After translation to a common coordinate system, the field patterns of neighboring patches are aggregated to obtain the radiation pattern of a larger patch. This sequence of operations is repeated until the pattern of the whole radiating surface is obtained.

This algorithm provides orders of magnitude acceleration of the PO integral evaluation, because it reduces the computation complexity from $O\left(N^{4}\right)$ to $O\left(N^{2} \log N\right)(N=k R$, with $R$ the radius of the smallest 
circumscribing sphere and $k$ the wavenumber). The MLPO algorithm achieves a computational efficiency similar to that of the FFT-based algorithms, while not being subject to their limitations (uniform sampling on planar apertures). The algorithm has been further developed in [5] to analyze dual reflector antennas.

The following extensions of the original MLPO scheme have been developed to facilitate the analysis of multi-reflector antenna systems such as the RATAN-600 antenna:

a) Near field formulation for field interpolation and aggregation, when successive reflectors are not in the far field of the previous one.

b) Generic multi-reflector computational scheme for sequential propagation between the reflectors and aggregation of the radiation patterns produced by all system components.

c) Adaptive hierarchical decomposition of radiating surfaces along dimensions that are substantially different in their sizes.

These extensions make the MLPO algorithm adaptive to arbitrary shaped multi-reflector antennas of very large dimensions.
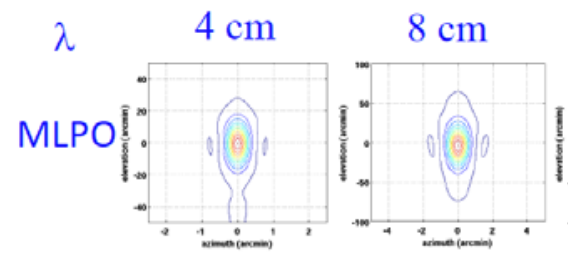

$48 \mathrm{~cm}$
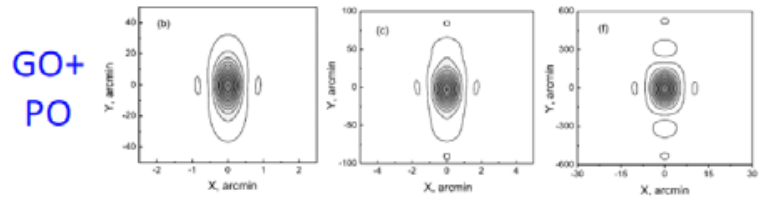

Fig. 4. Antenna radiation patterns computed using MLPO and geometrical optics ray tracing followed by aperture integration.

\section{NUMERICAL RESULTS}

Application of the MPLO algorithm to RATAN-600 radio telescope was considered in [6]. Computation of the RATAN-600 radio telescope power beam patterns at different wavelengths with allowance for diffraction effects in the southern sector with flat-reflector mode [7] are presented for comparison with the MLPO results in Fig. 4.

Multi-beam radiation patterns of the RATAN-600 radio telescope obtained for the new operation modes using the adaptive MLPO algorithm will be presented at the conference in comparison with these calculated by the combined GO-aperture integration method.

\section{REFERENCES}

[1] N. L. Kajdanovskij, "Automatic radio source tracking in observations with variable profile antenna," Astrophyz. Issled. (Izvestia SAO), vol. 7, pp. 214-222, 1975.

[2] E. K. Majorova, V. B. Khaikin, "Computation of RATAN-600 characteristics in operation as a "radioSchmidt telescope'," Astrophyz. Issled. (Izvestia SAO), vol. 48, pp. 133-149, 1999.

[3] V. B. Khaikin, E. K. Majorova, I. G. Efimov, O. A. Victorov, "Long time cosmic source tracking at RATAN-600 in 'radio-Schmidt' mode," Astronomy \& Astrophysics Transactions, no. 3-4, pp. 596-611, 2000.

[4] A. Boag and C. Letrou, "Multilevel Fast Physical Optics algorithm for radiation from non-planar apertures," IEEE Trans. Antennas Propagat., vol. 53, no. 6, pp. 2064-2072, June 2005.

[5] C. Letrou and A. Boag, "Analysis of very large dualreflector antennas using Multilevel Physical Optics algorithm," ICEAA 2007: International Conference on Electromagnetics in Advanced Applications, Turin, Italy, Sept. 2007.

[6] M. K. Lebedev, V. B. Khaikin, A. Boag, and C. Letrou, "Optical and diffraction simulation techniques for large multibeam reflector, MSMW'10: Seventh IEEE International Kharkov Symposium on Physics and Engineering of Microwaves, Millimeter and Submillimeter Waves, Kharkiv, Ukraine, pp. 1-3, June 21-26, 2010.

[7] E. Majorova, Computation of the RATAN-600 radio telescope power beam pattern with allowance for diffraction effects in the southern sector with flatreflector mode, Astrophys. Bull. 66 (2011) 215-232. 